\title{
Abdominal rectus muscle pyomyositis: Report of a case and review of the literature
}

\author{
Tilemachos Fountoukis, ${ }^{1}$ \\ Nikolaos Tsatsanidis, ${ }^{1}$ \\ Maria Tilkeridou, ${ }^{1}$ \\ Ioannis Konstantinou, ${ }^{2}$ Pantelis Fytas, ${ }^{3}$ \\ Ioannis Skandalos ${ }^{1}$ \\ ${ }^{1}$ Surgical Department, ${ }^{2}$ Radiology \\ Department, ${ }^{3}$ Microbiology Department, \\ General Hospital Agios Pavlos, \\ Thessaloniki, Greece
}

\begin{abstract}
Pyomyositis is an uncommon primary bacterial infection of skeletal muscles, usually caused by Staphylococcus aureus. Predisposing factors for pyomyositis include immunodeficiency, trauma, injection drug use, concurrent infection and malnutrition. The diagnosis, staging of the disease and differential diagnosis are established by ultrasound, CT and MRI Treatment involves surgical drainage and antibiotic therapy. We report a case of abdominal rectus muscle pyomyositis, which constitutes, as far as we know, the second reported in bibliography, while Prevotella disiens is firstly reported as causative agent.
\end{abstract}

\section{Introduction}

Pyomyositis is an uncommon primary bacterial infection of skeletal muscles, usually caused by Staphylococcus aureus. ${ }^{1}$ It is presumably hematogenous in origin, not associated with contiguous infection of the skin, bone or soft tissue, ${ }^{2}$ and often leads to abscess formation. ${ }^{3}$ Pyomyositis is primarily an infection of the tropics, although it has been recognized in temperate climates with increasing frequency. ${ }^{3}$ Most patients with tropical pyomyositis are otherwise healthy without underlying comorbidities, while most patients in temperate regions are immunocompromised or have other serious underlying conditions. The disease is usually diagnosed late and for this reason it carries high morbidity and significant mortality. ${ }^{4,5}$ Differential diagnosis and staging are established by ultrasound, CT and MRI. The agent responsible can be isolated from blood cultures. Treatment relies on antibiotic therapy and surgical drainage. We report a case of abdominal rectus muscle pyomyositis, which constitutes, as far as we know, the second reported in bibliography. ${ }^{6}$

\section{Case Report}

An 81-year-old patient was admitted to our hospital emergency department with a gradually increasing, during the last 15 days, painful swelling in the lower abdomen at the right abdominal rectus muscle (Figure 1). Patient reported that he initially felt pain after strenuous muscle activity (lifting of a heavy object). The inflamed swelling was accompanied with general symptoms of weakness, fever and chills. The patient's medical history included only hypertension. Hemodynamic findings at the time of admission were: arterial blood pressure $60 / 40 \mathrm{~mm} / \mathrm{Hg}$, temperature $37.4^{\circ} \mathrm{C}$, heart rate $125 / \mathrm{min}, \mathrm{pH} 7.45, \mathrm{pO}^{2} 98.7 \mathrm{mmHg}$, $\mathrm{pCO}^{2} 26.0 \mathrm{~mm} \mathrm{Hg}$, Lactic Acid 1.3 mmol/L, HCO3- 20.9 mmol/L, Actual Base Excess (ABE) - $4.3 \mathrm{mmol} / \mathrm{L}$. Additional blood tests revealed Hct 37\%, leukocytes $26.800 / \mathrm{mm}^{3}, 96.5 \%$ granulocytes, platelets $317.000 / \mathrm{mm}^{3}$, blood glucose $197 \mathrm{mg} / \mathrm{Dl}$, urea $70 \mathrm{mg} / \mathrm{dL}$, creatinine $1.29 \mathrm{mg} / \mathrm{dL}, \mathrm{K}^{+}$ $3.8 \mathrm{mmol} / \mathrm{L}, \mathrm{Na}^{+} 128 \mathrm{mmol} / \mathrm{L}, \quad B l o o d$ (Serum) Glutamic-Oxaloacetic Transaminase (SGOT) 19 IU/L, Blood (Serum) Glutamic Pyruvic Transaminase (SGPT) 16 IU/L. With clinical suspicion of incarcerated Spigelian hernia, the patient underwent abdominal and chest $\mathrm{x}$-ray investigation, which were normal, and an abdominal CT scanning which revealed an abscess, with contrast agent-induced enhancing of the capsule as well as the internal diaphragms, inside the right abdominal rectus muscle below the umbilicus (Figure 2). Due to the diagnosis of abdominal rectus muscle pyomyositis, the patient underwent a surgical drainage of the abscess.

In pus culture, Streptococcus constellatus and Prevotella disiens were isolated. The initial empiric antibiotic therapy was modified according to the results of the antibiogram (Table 1) and Amoxycillin/Clavunanic acid was administered orally, $1 \mathrm{~g}$ twice per day, for an additional 3 weeks. The postoperative course was uneventful.

\section{Discussion}

Pyomyositis is an uncommon primary acute bacterial infection of skeletal muscles, 1985 by Scriba, ${ }^{7}$ as an endemic disease in the tropics. Pyomyositis is endemic in tropical areas affecting mainly young people and children while it is uncommon in non tropical areas affecting mainly adults and elderly patients. ${ }^{8}$ Trauma has a crucial role in development of pyomyositis, as it is found in $25-40 \%$ of the cases. ${ }^{9}$ The muscles
Correspondence: Fountoukis Tilemachos, Surgical Department, General Hospital Agios Pavlos, Thessaloniki, Greece.

Tel.: +30.6947002721.

E-mail: fountoukismd@gmail.com

Key words: abdominal rectus muscle, drainage, myonecrosis, Prevotella disiens, pyomyositis, Streptococcus constellatus.

Contributions: the authors contributed equally.

Conflict of interest: the authors declare no potential conflict of interest.

Funding: none.

Received for publication: 4 December 2017. Revision received: 22 January 2018.

Accepted for publication: 29 January 2018.

This work is licensed under a Creative Commons Attribution-NonCommercial 4.0 International License (CC BY-NC 4.0).

(C) Copyright T. Fountoukis et al., 2018

Licensee PAGEPress, Italy

Infectious Disease Reports 2018; 10:7522

doi:10.4081/idr.2018.7522

most commonly affected include the extensor femoral muscle group (mainly quadriceps femoris), the outer hip muscles (namely the gluteus muscles) and the inner hip muscles, especially the psoas as well as the iliacus muscle..$^{5,10}$ The abdominal rectus muscle pyomyositis is very rare and only one other case is referred in medical literature. ${ }^{6}$ In our case, this pathological condition could have been caused by hematogenous infection of an abdominal rectus muscle hematoma, as the patient correlated the initial pain at this location with strenuous muscle activity - lifting of a heavy object. Rectus sheath hematoma is a rare clinical condition that may occur after a spontaneous or a posttraumatic rupture of the epigastric vessels or the muscle fibers of the rectus abdominis muscle. ${ }^{11}$ When the hematoma is located above the semilunar line of Douglas it is usually limited, because of the existence of the posterior wall of the sheath. If it is located below this line, it can result in peritoneal infiltration or extension to the contralateral side, becoming bilobular. ${ }^{12,13}$

Predisposing factors to pyomyositis are diabetes mellitus, alcohol abuse, parenteral drug abuse, HIV infection, cancer, trauma and hematologic diseases. S. aureus is the most common causative organism isolated from blood or pus cultures..,14,15 Other, rarely, isolated germs are Streptococcus group bacteria, Escherichia coli, 
Salmonella species, Mycobacterium tuberculosis and other, as well as its combination. ${ }^{6,16,17}$ In our case, as referred above, a traumatic injury due to strenuous muscle activity was most likely the main predisposing factor. Streptococcus constellatus and Prevotella disiens were identified as the causative agents from blood cultures. This is the first case of Prevotella disiens reported in the literature.

$S$. constellatus belongs to the $S$. anginosus group (milleri group) along with $S$. intermedius and $S$. anginosus. These organisms are part of the human oropharyngeal microbiome and may be isolated from the throat, the nasopharynx, and the gingival crevices. They are also found in the gastrointestinal tract and in the vagina. S. intermedius, S. constellatus, and S. anginosus are recognized for their propensity to cause purulent tissue abscesses, intra-abdominal infections, pulmonary infections, bacteraemia, CNS infections, oral infections, and endocarditis. Bacteremia is usually attributable to a focus of infection within the gastrointestinal or upper respiratory tract. Suppurative metastatic abscesses are a significant complication of bacteremia, with S. intermedius and $S$. constellatus being more often associated with abscess formation than $S$. anginosus. ${ }^{18}$

Genus Prevotella are gram-negative, obligate anaerobic bacteria that constitute a major part of the normal flora of the mouth, gastrointestinal tract, and female genital tract and consist of pigmented and nonpig- mented species. $P$. disiens and $P$. bivia (previously called Bacteroides) are two very common members of the non pigmented Prevotella and are important in obstetric and gynecologic infections. P. bivia and P. disiens have been isolated from blood, head and neck infections, GU tract infections in both males and females, and other sites of infection. P. disiens is commonly isolated from the urogenital tract. ${ }^{19}$ Isolates of $P$. bivia, P. disiens, P. buccae, P. denticola, and P. nigrescens have been found in bloodstream infections. ${ }^{20}$

Pyomyositis usually presents as a subacute infection of a skeletal muscle, but may also present as a diffuse inflammatory or a rapidly progressing myonecrotic process as in our case. In the first (invasive) stage, the patient presents with muscle pain, pyrexia, and often weakness and the lab tests usually reveal mild leukocytosis, elevated inflammatory markers (e.g. CRP, ESR) and occasionally anemia. The second stage is characterized by marked oedema of the affected muscle and the patient presents signs of localized inflammation such as tenderness. In the third stage the systemic manifestations are severe; indeed our patient presented with a sepsis syndrome.

Toxic shock caused by a non-toxin producing strain of staphylococcus aureus and coma may develop..$^{10,21}$

$\mathrm{X}$-rays are usually unremarkable. The diagnosis is established with an US imaging and verified by a CT scanning, ${ }^{8,10,13}$ which assessed the exact location and extension of

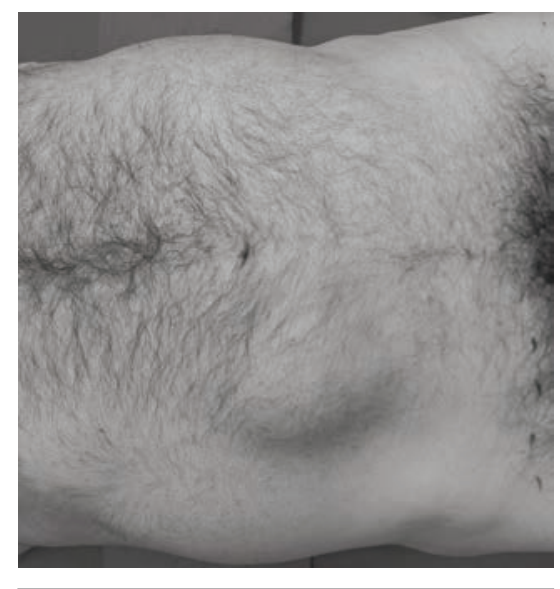

Figure 1. Swelling in the lower abdomen at the right abdominal rectus muscle, below the umbilicus.

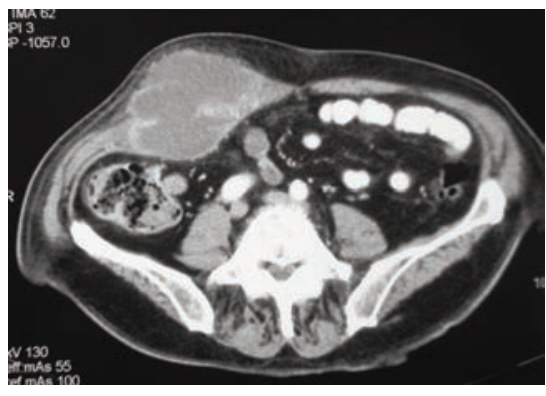

Figure 2. Abdominal computed tomography: abscess with diaphragms, inside the right abdominal rectus muscle below the umbilicus.

Table 1. Identification for both isolates (performed on automated BIOMÉRIEUX VITEK® 2 SYSTEM). Antimicrobial susceptibility test (AST) was performed: A) for isolate No 1: Streptococcus constellatus spp. constellatus, on automated BIOMÉRIEUX VITEK® 2 SYSTEM; B) for isolate No 2: Prevotella disiens, on SENSITITRETM ANAEROBE PLATE FORMAT. Susceptibility to ampicillin, amoxicillin, Amoxicillin-clavulanic acid was inferred from susceptibility to benzylpenicillin. $(<0.06$ : S) (EUCAST Clinical Breakpoint Table v. 5.0)

\section{Streptococcus constellatus spp. constellatus} MIC

\section{Cat}

Amoxicillin

Amoxicillin/clavulanic acid

\begin{tabular}{|c|c|c|c|c|}
\hline HIIIOXICinmI/Ciavu & & $\mathrm{S}$ & & $\mathrm{J}$ \\
\hline Ampicillin & $\leq 0.25$ & S & & S \\
\hline Ampicillin/Sulbac & & S & & \\
\hline Benzylpenicillin & $\leq 0.06$ & S & $<0.06$ & S \\
\hline Cefotaxime & 0.25 & S & & \\
\hline Cefoxitin & & & $<1$ & $\mathrm{~S}$ \\
\hline Ceftriaxone & 0.25 & S & & \\
\hline Clindamycin & $\leq 0.25$ & S & $<0.25$ & S \\
\hline Erythromycin & $\leq 0.12$ & S & & \\
\hline Imipenem & & & $<0.12$ & S \\
\hline Levofloxacin & $\leq 0.25$ & S & & \\
\hline Metronidazole & & & $<0.5$ & $\mathrm{~S}$ \\
\hline Piperacillin/Tazol & & S & & S \\
\hline Tetracycline & 1 & $\mathrm{~S}$ & & \\
\hline Vancomycin & 0.25 & S & & \\
\hline
\end{tabular}


the abscess and the condition of the rectus abdominis muscle. Differential diagnosis includes inflammatory abdominal disorders or abdominal tumors, ${ }^{22}$ and other disorders of the abdominal wall, ${ }^{23}$ such as hematoma, tumors (desmoid, neurinomas, sarcomas) and abscesses due to perforated diverticula. In our case, the diagnosis was confirmed with a CT scanning.

Pyomyositis therapy consists of surgical or radiologically-guided drainage of the abscesses, accompanied with broad-spectrum intravenous antibiotic therapy and management of accompanying diseases ${ }^{10,15,24}$ In our case, open drainage of the abscess under local anaesthesia was the therapy of choice, because the abdominal rectus muscle is located immediately beneath the skin, without any other tissues in between. Hyperbaric oxygen therapy (HBOT) in the treatment of pyomyositis, is referred only in one case of iliacus muscle pyomyositis with contribution, as an adjunct to antibiotic therapy, to the favorable outcome of the case. ${ }^{25}$ Antibiotic therapy optimal duration has not been established. In our case the surgical drainage of abdominal rectus sheath abscess was done under antibiotic therapy simultaneous treatment. The antibiotic therapy was modified later in accordance with the result of pus culture.

\section{Conclusions}

In conclusion, pyomyositis of the abdominal rectus muscle is a very rare condition, but it must be suspected as a possible cause of acute abdominal rectus muscle pain, marked oedema, localized inflammation and pyrexia. Administration of antibiotics and early drainage of the abscess remain the cornerstone of therapy.

\section{References}

1. Burdette SD, Watkins RR, Wong KK et al. Staphylococcus aureus pyomyositis compared with non-Staphylococcus aureus pyomyositis. J Infect 2012;64:507-12.

2. Chen WS Wan YL. Iliacus pyomyositis mimicking septic arthritis of the hip joint. Arch Orthop Trauma Surg 1996;115:233-5.

3. Small LN, Ross JJ. Tropical and temperate pyomyositis. Infect Dis Clin North Am 2005;19:981-9.

4. Drosos G. Pyomyositis. A literature review. Acta Orthop Belg 2005;71:9-16.

5. Lee $\mathrm{MH}$, Byon $\mathrm{HJ}$, Jung $\mathrm{HJ}$, et al. Pyomyositis of the iliacus muscle and pyogenic sacroiliitis after sacroiliac joint block. A case report. Korean J Anesthesiol 2013;64:464-8.

6. Pare JR, Schechter-Perkins EM. Temperate pyomyositis: a pain in the belly. Am J Emerg Med 2015;33:306.

7. Scriba J. Beitrag zur aetiologie der myositis acuta. Dtsch Ztschr Chir 1885;22:497-502 .

8. Peckett WR, Butler-Manuel A, Apthorp LA. Pyomyositis of the iliacus muscle in a child. J Bone Joint Surg $\mathrm{Br}$ 2001;83:103-5.

9. Comegna L, Guidone PI, Prezioso G, et al. Pyomyositis is not only a tropical pathology: a case series. J Med Case Rep 2016;10:372.

10. Agarwal N, Aroor S, Saini P, et al. Pyomyositis: Are We Missing the Diagnosis? Surg Infect (Larchmt) 2016;17:615-21.

11. Zainea GG, Jordan F. Rectus sheath hematomas: their pathogenesis, diagnosis, and management. Am Surg 1988;54:630-3.

12. Fitzgerald JE, Fitzgerald LA, Anderson FE, Acheson AG. The changing nature of rectus sheath haematoma: case series and literature review. Int J Surg 2009;7:150-4.

13. Skandalos IK, Kalfadis SC, Parpoudi $\mathrm{SN}$, et al. Rectus sheath hematoma: a diagnostic and therapeutic dilemma. Med Sci Rev 2015;2:19-23.

14. Karmazyn B, Kleiman MB,
Buckwalter K, et al. Acute pyomyositis of the pelvis: the spectrum of clinical presentations and MR findings. Pediatr Radiol 2006;36:338-43.

15. Chiu SK, Lin JC, Wang NC, et al. Impact of underlying diseases on the clinical characteristics and outcome of primary pyomyositis. J Microbiol Immunol Infect 2008;41:286-93.

16. Simopoulou T, Varna A, Dailiana Z, et al. Tuberculous pyomyositis: a reemerging entity of many faces. Clin Rheumatol 2016;35:1105-10

17. Vigil KJ, Johnson JR, Johnston BD, et al. Escherichia coli Pyomyositis: an emerging infectious disease among patients with hematologic malignancies. Clin Infect Dis 2010 1;50:374-80

18. Koneman's Color Atlas and Textbook of Diagnostic Microbiology. 2017;13:765-6.

19. Koneman's Color Atlas and Textbook of Diagnostic Microbiology. 2017;16: 999-1000,1032-5.

20. Simmon KE, Mirrett S, Reller LB, et al. Genotypic diversity of anaerobic isolates from bloodstream infections. J Clin Microbiol 2008;46:1596-601.

21. Chiedozi LC. Pyomyositis. Review of 205 cases in 112 patients. Am J Surg 1979;137:255-9.

22. Alla VM, Karnam SM, Kaushik M, Porter J. Spontaneous rectus sheath hematoma. West J Emerg Med 2010;11:76-9.

23. Klingler PJ, Wetscher G, Glaser K, et al. The use of ultrasound to differentiate rectus sheath hematoma from other acute abdominal disorders. Surg Endosc 1999;13:1129-34.

24. Palacio EP, Rizzi NG, Reinas GS, et al. Open drainage versus percutaneous drainage in the treatment of tropical pyomyositis. Prospective and randomized study. Rev Bras Ortop 2015;45:260-8.

25. Skandalos I, Kirmizis I, Kampranis I, et al. Pyomyositis of the iliacus muscle - a diagnostic and therapeutic dilemma. Hel Jour Surg 2014;86:216-20. 